\title{
In vitro and computational studies of transdermal perfusion of nanoformulations containing a large molecular weight protein
}

\author{
Madalena Martins a ${ }^{\text {, Nuno G. Azoia }}{ }^{a}$, Artur Ribeiro ${ }^{a}$, Ulyana Shimanovich ${ }^{\mathrm{b}}$, \\ Carla Silva ${ }^{a}$, Artur Cavaco-Paulo ${ }^{a, *}$ \\ a IBB - Institute for Biotechnology and Bioengineering, Centre of Biological Engineering, University of Minho, 4710-057 Braga, Portugal \\ ${ }^{\mathrm{b}}$ Department of Chemistry, University of Cambridge, Lensfield Road, Cambridge, CB2 1EW, United Kingdom
}

\section{A R T I C L E I N F O}

\section{Article history:}

Received 9 January 2013

Received in revised form 13 February 2013

Accepted 14 February 2013

Available online 4 March 2013

Keywords:

Protein

Surfactant

Nanodispersion

Transdermal perfusion

Hydrophilic macromolecules

\begin{abstract}
A B S T R A C T
Transdermal perfusion of a large protein is reported for the first time, using a nanoemulsion of bovine serum albumin ( $66 \mathrm{kDa}$ ) of $160 \mathrm{~nm}$ prepared by a solid-in-oil (S/O) process. Molecular dynamics simulations confirmed skin permeation by these formulations, with integration of the protein into the lipid bilayers. These results demonstrate the real possibility of delivering large proteins transdermally for a range of medical and cosmetic applications.
\end{abstract}

(C) 2013 Elsevier B.V. All rights reserved.

\section{Introduction}

Transdermal perfusion of large hydrophilic molecules such as globular proteins, remains a challenging task. This is mainly due to the lipophilic nature of skin's barrier [1-3]. The barrier function of mammalian skin is mainly attributed to the stratum corneum (SC), the outer protective layer. This consists of flat, dead cells filled with keratin fibers (corneocytes) and surrounded by lipid bilayers that confer impermeability to relatively large compounds (molecular weight over $500 \mathrm{Da}$ ) [4,5]. Several lipids can be found in the SC of human's skin, the most important being ceramides, free fatty acids, cholesterol and cholesterol sulfate. In comparison with other human cells/tissues, human SC contains extremely complex ceramides (CERs), consisting of at least 11 classes several fatty acids and sphingoid moieties on SC [6-8]. These classes are combinations of nonhydroxy, $\alpha$-hydroxy, $\omega$-hydroxy and esterified $\omega$-hydroxy fatty acids containing dihydrosphingosines, sphingosines, phytosphingosines and 6-hydroxy-sphingosines. The endogenous free fatty acid fraction of SC barrier lipids are in essence exclusively comprised of saturated long-chain free fatty acids. This fraction was characterized as a very stable population (low inter individual peak variation) dominated by saturated lignoceric acid (C24:0-39 mol\%) and hexacosanoic acid (C26:0-23 mol\%) [9]. It is therefore expected

\footnotetext{
* Corresponding author. Tel.: +351 253604 400; fax: +351 253604429

E-mail address: artur@deb.uminho.pt (A. Cavaco-Paulo).
}

that hydrophilic macromolecules, such as proteins or peptides, will only pass through the SC barrier if incorporated into dispersion formulations with an external layer of a hydrophobic moiety [10].

The use of S/O dispersions is reported to mediate the skin permeability of proteins of $6 \mathrm{kDa}$ after $48 \mathrm{~h}$ of treatment. Larger proteins of 27 and $40 \mathrm{kDa}$ (nanodispersions with sizes of 210-260 nm) gave unsatisfactory perfusion results [11-14]. Other authors have used $\mathrm{S} / \mathrm{O}$ dispersions of $190 \mathrm{~nm}$ to improve the transdermal delivery of large proteins for skin vaccination. In this case however, skin perfusion was only achieved using an external photodermal effect [12]. These results suggest, that in order to achieve the successful skin perfusion of a larger target protein, the sphere size of the hydrophobic nanoemulsion should be reduced. In order to test this hypothesis, we adapted and further optimized a S/O methodology to incorporate a large protein of $66 \mathrm{kDa}$, namely BSA (bovine serum albumin) and achieve oil-based nanodispersions with an average sphere size of $162 \mathrm{~nm}$.

\section{Materials and methods}

\subsection{Materials}

Nanodispersions preparation was performed using AlbuminFITC, chloroform and isopropyl myristate purchased from Sigma-Aldrich (Spain). The sucrose ester (SP10-C) was supplied by Sisterna (Netherlands). The transdermal studies were performed using pig skin from slaughtery and kindly supplied by Matadouro 


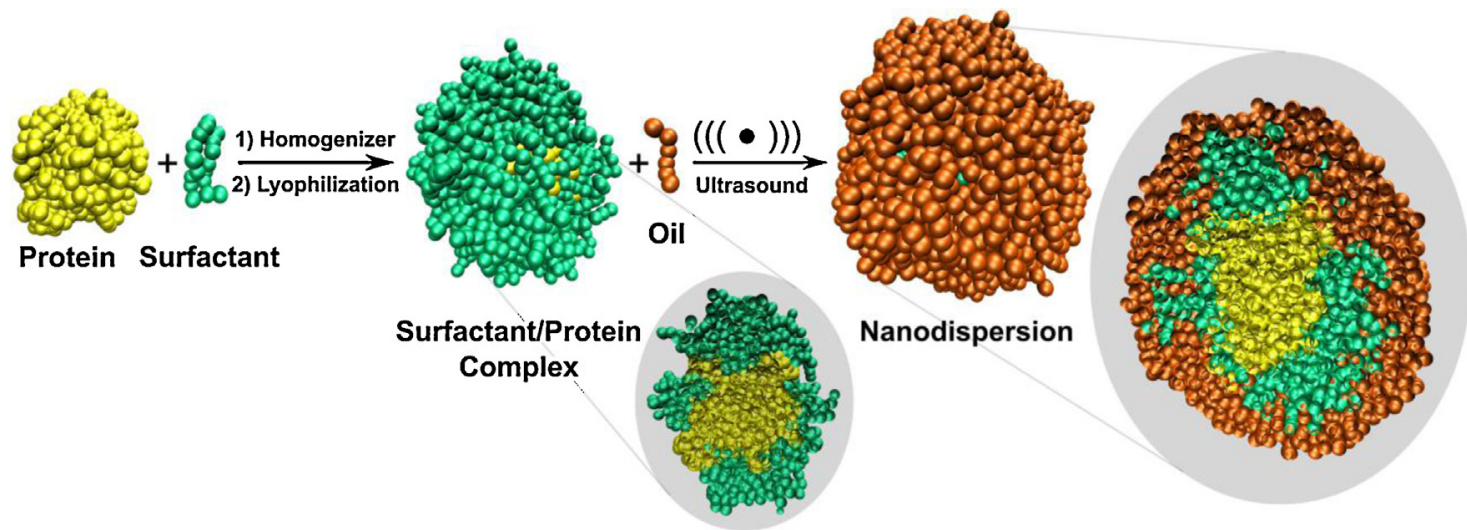

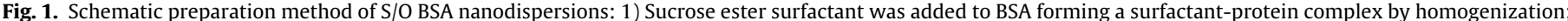

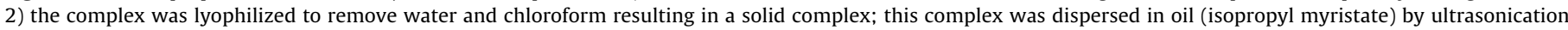
forming the final S/O nanodispersion.

Central de Entre Douro e Minho (Portugal). For histology studies the Cryoprotectant Tissue-Tek Oct compound was purchased from Sakura (Netherland) and the Thermo Scientific Shandon ImmuMount was purchased from Fisher Healthcare (UK). All other reagents were from laboratory grade without any further purification.

\subsection{Solid-in-oil nanodispersions preparation}

Albumin-FITC nanodispersions were prepared using a solid-inoil technique based on three different stages: (1) an initial complex between sucrose ester surfactant (SP10-C) $(25 \mathrm{mg} / \mathrm{mL})$ dissolved in chloroform and a protein (BSA-FITC) $(0.5,1,5$ and $10 \mathrm{mg} / \mathrm{mL})$ was formed utilizing high pressure homogenization (EmulsiFlex ${ }^{\circledR}-\mathrm{C} 3$ supplied from Avestin, Inc., Canada) at 1000 bar; (2) a lyophilization step was then performed in order to eliminate any water and chloroform and thereby yield a solid surfactant-protein complex; (3) the resulting lyophilized complex was then dispersed in $15 \mathrm{~mL}$ of isopropyl myristate by ultrasonication at $20 \mathrm{kHz}$ (Sonics \& Materials Vibracell CV33, fitted with a $3 \mathrm{~mm}$ diameter titanium micro-tip) to form the final S/O nanodispersion (Fig. 1). Fig. 2 presents the final physical appearance of bovine serum albumin-FITC in the absence and in the presence of sucrose ester surfactant (Fig. 2A) as well as its chemical structure.

\subsection{Solid-in-oil nanodispersions characterization}

\subsubsection{Size distribution}

Particle size distribution and polydispersity index (for each nanodispersion the average size was measured in triplicate) of the solid-in-oil nanodispersions was determined at $25 \pm 0.1^{\circ} \mathrm{C}$ by photon correlation spectroscopy (PCS) using a Malvern Zetasizer NS (Malvern Instruments). Since emulsions can suffer aggregation over
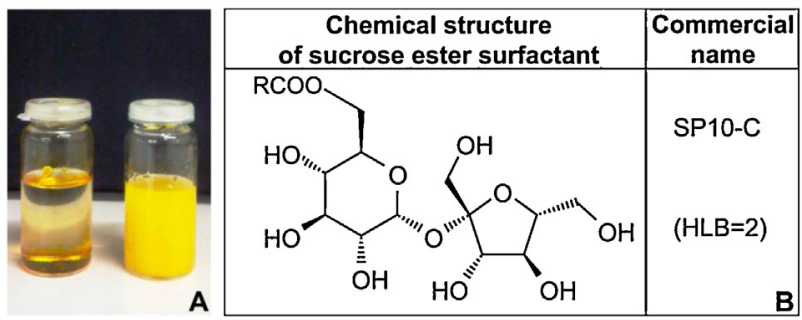

Fig. 2. (A) Physical appearance of final S/O nanodisperision without sucrose ester (Left) and final S/O nanodispersion with sucrose ester (Right); (B) Chemical structure of sucrose ester surfactant. time, the stability of produced nanodispersions was analyzed. The stability was evaluated by measurements in terms of particle size and polydispersity index.

\subsubsection{Scanning transmission electron microscopy (STEM)}

The morphological characterization of nanodispersions was conducted using transmission electron microscopy (STEM) technique in an electron microscope model LEICA S360 with a backscattered and secondary electrons detector, at 50,000× magnification. The samples were placed on copper grids with carbon film for STEM observation.

\subsubsection{Nanodispersions production efficiency}

The efficiency of final nanodispersions production was determined by separation of the formulated protein from the free Albumin-FITC. For this, the nanodispersions were centrifuged at $(15,000 \times g ; 30 \mathrm{~min})$ using Amicon tubes $(15 \mathrm{kDa})$. The amount of free Albumin-FITC was determined in the supernatant by the Lowry method protein assay (Sigma), measuring the absorbance by spectrophotometry (Microplate Reader Synergy ${ }^{\mathrm{TM}}$ MX, Bioteck Instruments) at $750 \mathrm{~nm}$ [15]. Each sample was assayed in triplicate. The concentration of protein was determined by a calibration curve. The nanodispersions efficiency was calculated as follows:

efficiency $(\%)=\frac{[\text { protein }]_{i}-[\text { protein }]_{f}}{[\text { protein }]_{i}} \times 100$

where $[\text { protein }]_{i}$ is the initial protein concentration and [protein $]_{f}$ is the final protein concentration in the supernatant after formulation.

\subsection{In vitro permeation studies}

In vitro permeation of $\mathrm{S} / \mathrm{O}$ nanodispersions was performed using an appropriate apparatus (V-Series Stirrers for Franz Cells) (PermeGear, USA). Pig skin sections were placed between two compartments: the donor and the receptor compartment. Donor compartment was filled with $300 \mu \mathrm{l}$ of formulation and the receptor compartment was filled with $5 \mathrm{~mL}$ of $0.01 \mathrm{M}$ phosphate buffered saline ( $\mathrm{pH} 7.4$ ). This receptor liquid was maintained at $37^{\circ} \mathrm{C}$ using a circulating water bath and was continually stirred with a small magnetic bar for uniform mixing. Aliquots were collected at predetermined time intervals ( $0 \mathrm{~h}, 2 \mathrm{~h}, 4 \mathrm{~h}, 6 \mathrm{~h}, 8 \mathrm{~h}, 24 \mathrm{~h}, 48 \mathrm{~h}$ ) and each volume removed was replaced with the same amount of fresh buffer. S/O nanodispersions concentration in the receptor compartment was determined by Lowry method. 


\subsubsection{Confocal microscopy studies}

The penetration extent and localization of BSA-FITC nanodispersions was made using confocal laser microscopy using a Zeiss LSM 510 confocal microscope. The image reconstructions were made using "Imaris" image analysis software.

\subsubsection{SDS-Page electrophoresis}

The procedure was performed using SDS-PAGE with a $12 \%$ of resolving gel and $4 \%$ of stacking gel. An aliquot of the samples, $16 \mu \mathrm{l}$ of protein, was electrophoresed on a SDS polyacrylamide gel for $4 \mathrm{~h}$ at $80 \mathrm{~V}$. Proteins were visualized using Coomassie blue staining which has a detection limit of $\sim 1 \mu \mathrm{g} /$ protein band. Molecular weight protein native was used to compare the electrophoretic mobility of nanodispersion and native albumin bovine.

\subsection{Molecular dynamics simulation}

The simulations were performed with GROMACS 4.0.7[16] package using the Martini force field [17]. The system size was chosen according to the minimum image convention taking into account a cut-off of $1.2 \mathrm{~nm}$. The bonds lengths were constrained with LINCS [18]. Non-bonded interactions were calculated using a twin-range method, with short and long range cut-offs of 0.9 and $1.2 \mathrm{~nm}$, respectively. Neighbor searching was carried out up to $1.2 \mathrm{~nm}$ and updated every ten steps. A time step of integration of $20 \mathrm{fs}$ was used. A reaction field correction for the electrostatic interactions was applied using a dielectric constant of 15. Pressure control was implemented using the Berendsen barostat[19], with a reference pressure of $1 \mathrm{bar}, 3.0 \mathrm{ps}$ of relaxation time and isothermal compressibility of $3.0 \times 10-5$ bar $^{-1}$. Temperature control was set using the Berendsen thermostat [19] at $300 \mathrm{~K}$. Each component of the system was included in separated heat bath, with temperatures coupling constants of $0.30 \mathrm{ps}$. Five replica simulations of $475 \mathrm{~ns}$ in length were carried out using different initial velocities taken from a Maxwell-Boltzman distribution at $300 \mathrm{~K}$.

The lipid membrane was build using CELLmicrocosmos 2.2 package [20]. The composition of the membrane was based in previously reported values for young-normal skin samples [21] and in accordance with previously reported simulations [22]: ceramide-2, lignoceric acid, cholesterol and cholesterol sulphate [23]. Initially one bilayer was built and sodium ions were added to unsure that the system remains electrically neutral. This bilayer was replicated to build a double bilayer, representative of the stratum corneum lipid layer.

The protein microsphere model was constructed separately. In a first stage the protein and sucrose ester were added to a chloroform simulation box, allowing the formation of the protein-surfactant complex. The chloroform was then removed from the box and isopropyl myristate and water were added, originating the protein microsphere.

\section{Results and Discussion}

\subsection{Preparation and characterization of solid-in-oil nanodispersions}

The nanodispersions were made using a two phase high energy methodology utilizing homogenization and ultrasonication in which both high shear forces and cavitation impinge upon the BSA and the S/O [24]. Ultrasonication is utilized due to the inherent mechanisms of micro-stirring, compressions and decompressions that occur within the material [25]. The resulting heating and/or cavitation that also occurs contributes to the enhanced mass transfer and the ultimate formation of the nano scaled dispersions [26-28]. The homogenization phase of this process allows the formation of a homogeneous and stable surfactant-protein complex, which is then separated by lyophilization. The lyophilized powder is then dispersed in oil (solid-in-oil) using gentle ultrasonication. This methodology allowed to obtain a high efficiency nanodispersions production of $82 \%$.

In this work we aim to achieve small and stable nanodispersions for skin perfusion. To that end we have investigated the effects of protein concentration and the chemical nature of the sucrose ester surfactant on the size of the micelles. The size and the polydispersity of the emulsions was the decisive factor throughout the optimization of the formulation using the S/O method. Several sucrose ester surfactants with different HLB values (hydrophiliclipophilic balance) were tested and the best results were achieved for SP10-C with a HLB=2 (data not shown). Higher molar ratios of surfactant over protein were found to yield smaller dispersions (Fig. 3A). A SE/BSA molar ratio of 13,200:1 was determined to be optimal in order to yield smaller dispersions. However, it seems that there is a lower threshold of this SE/BSA molar ratio of 2640:1 and it is required for the stability of the dispersions over 8 weeks. The results obtained suggest an optimal value of molar ratio between the sucrose ester surfactant and BSA to yield a smaller nanodispersion in oil that is also stable and therefore amenable to storage.

The sizes were determined by scanning transmission electron microscopy (STEM) (Fig. 4B), and were consistent with the average diameter determined by PCS (Fig. 4A). The results observed
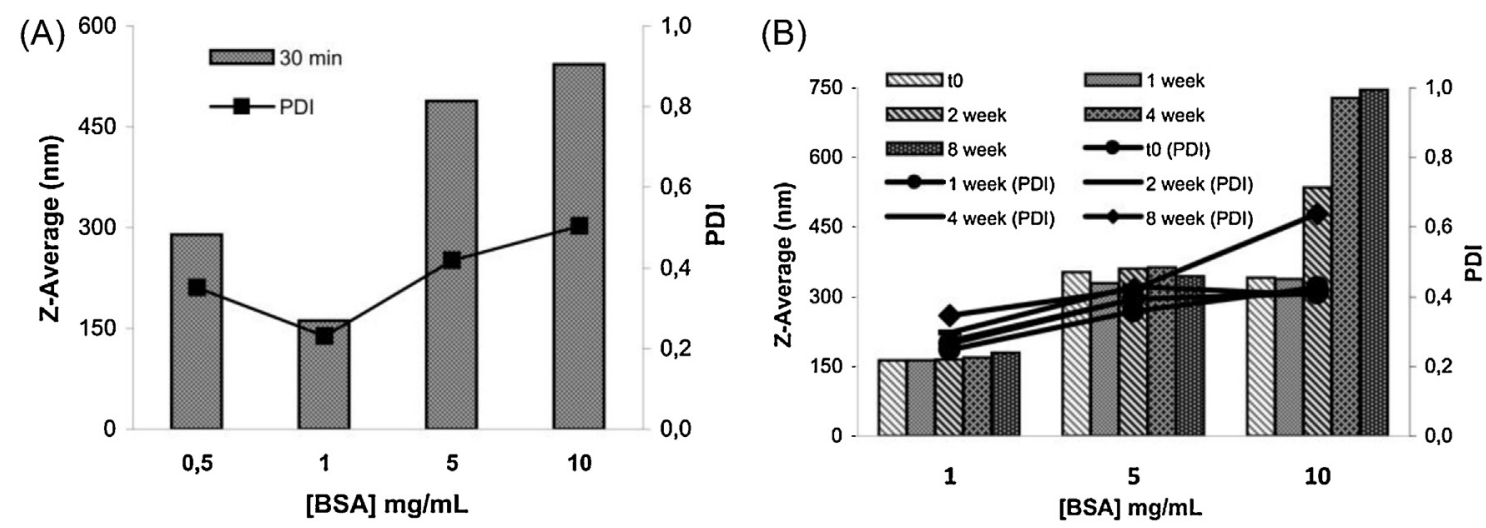

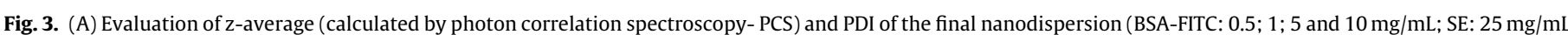

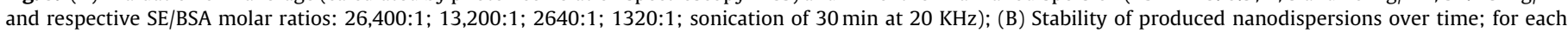
sample the average size was measured in triplicate. 

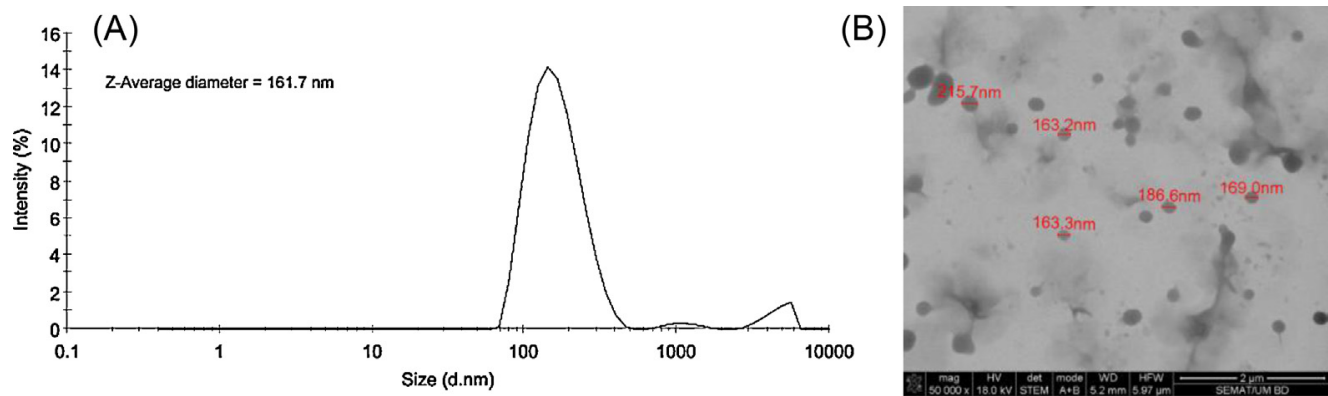

Fig. 4. (A) Size distribution obtained by PCS of S/O nanodispersion; (B) STEM microphotographs at $50000 \mathrm{x}$ magnification.

indicate that spherical nanodispersions were obtained with an average diameter of $160-180 \mathrm{~nm}$.

\subsection{In vitro permeation studies of solid-in-oil nanodispersions}

After successful preparation of the S/O BSA nanodispersions, in vitro permeation of BSA through pig skin was investigated using fluorescein isothiocyanate (FITC)-labeled BSA. Transdermal perfusion was assessed with the optimum candidate formulation obtained. A Franz diffusion cells system was used to assess pig skin penetration and the results were analyzed in order to determine the most efficacious formulation for pig skin penetration. The specimens of pig skin were provided by a local slaughterhouse. There is a limited availability of viable human skin for in vitro assessments due to ethical constraints restricting their harvest. As a consequence pig skin has been utilized in several studies and has proven to be an ideal animal model for human skin. There is considerable evidence that its barrier thickness $(66-72 \mu \mathrm{m})$ approaches that of human tissue $(70 \mu \mathrm{m})$ [29-37] and that the follicular structure of pig skin also resembles that of humans [37-39]. Furthermore, the vascular anatomy and collagen fiber arrangement within the dermis, as well as the relative content of both SC glycosphingolipids and ceramides are comparable between the domestic pig and humans [40].
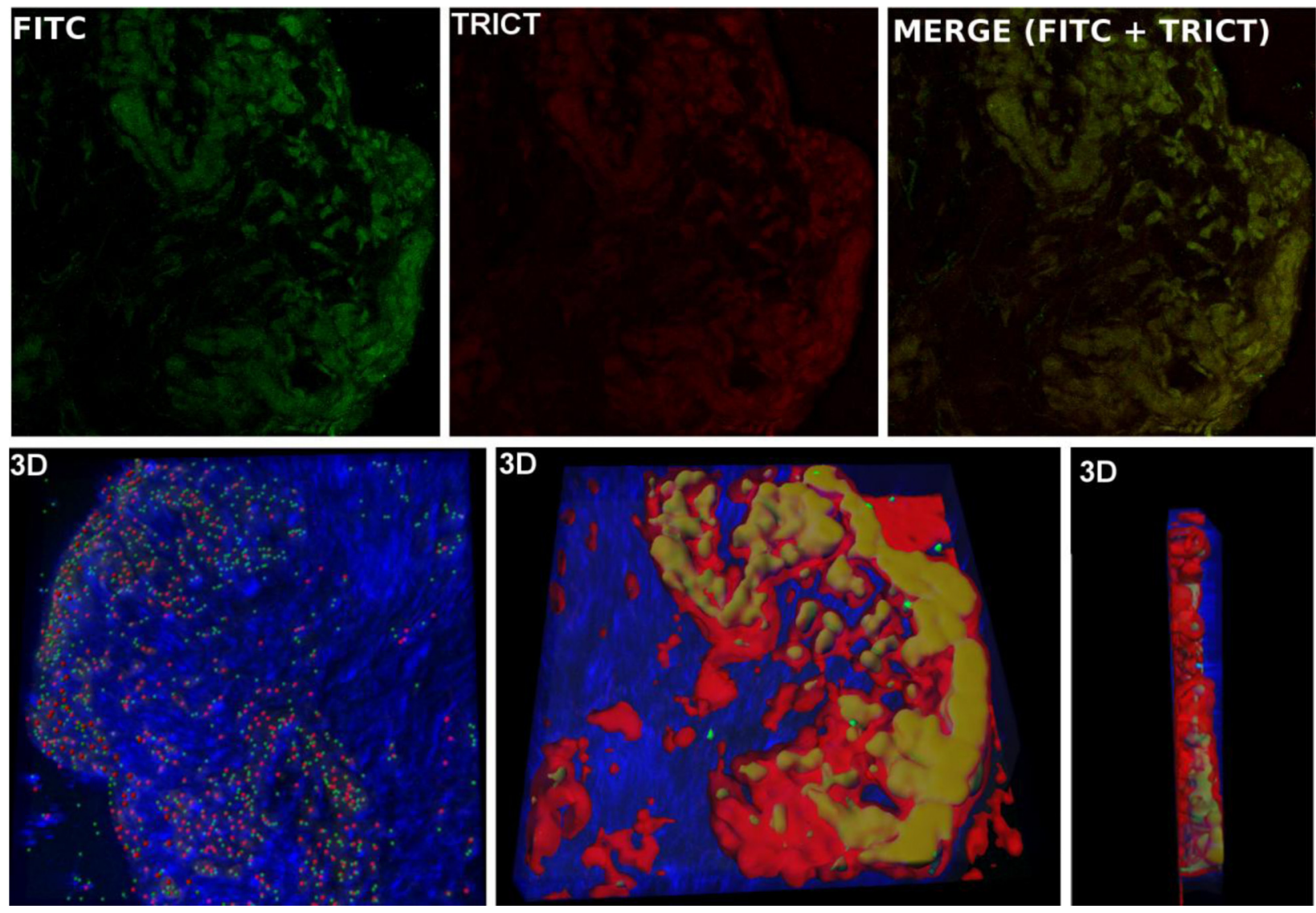

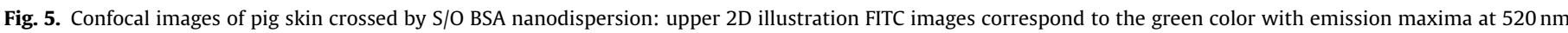

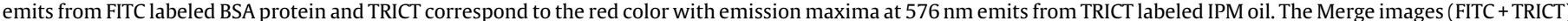

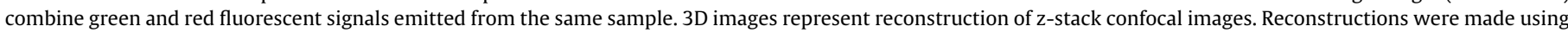

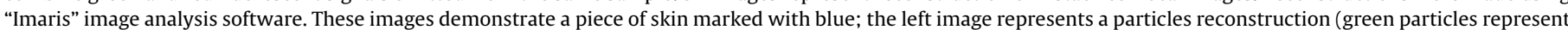

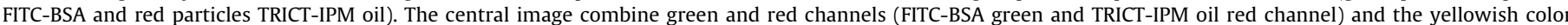

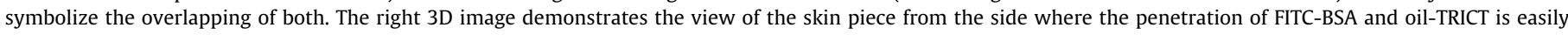
detected. (For interpretation of the references to color in this figure legend, the reader is referred to the web version of this article.) 
(A)

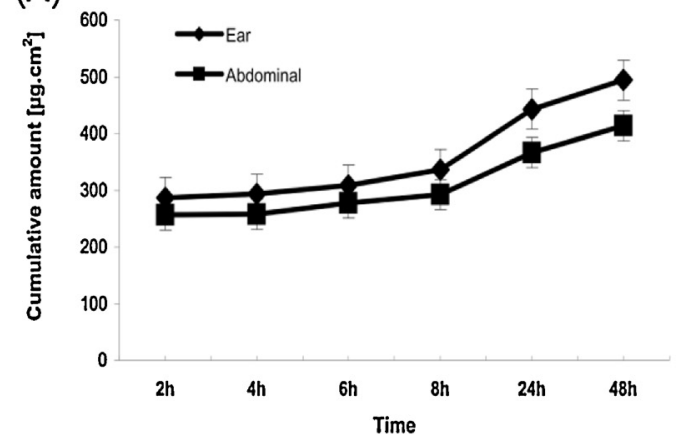

Cumulative nanodispersion permeated $=\mathbf{Q} \mathbf{t}$

$$
Q t=\operatorname{VrCt}+\sum_{i=0}^{t-1} V s C i
$$

Ct: Nanodispersion concentration of the receiver solution at each sampling time calculated by Lowry method;

Ci : Initial nanodispersion concentration calculated by Lowry method;

Vr: Volume of the receiver solution;

Vs: Volume of the sample.

The data are expressed as the cumulative nanodispersions permeation per unit of skin surface area, $\mathrm{Qt} / \mathrm{A}\left(\mathrm{A}=0,785 \mathrm{~cm}^{2}\right)$.
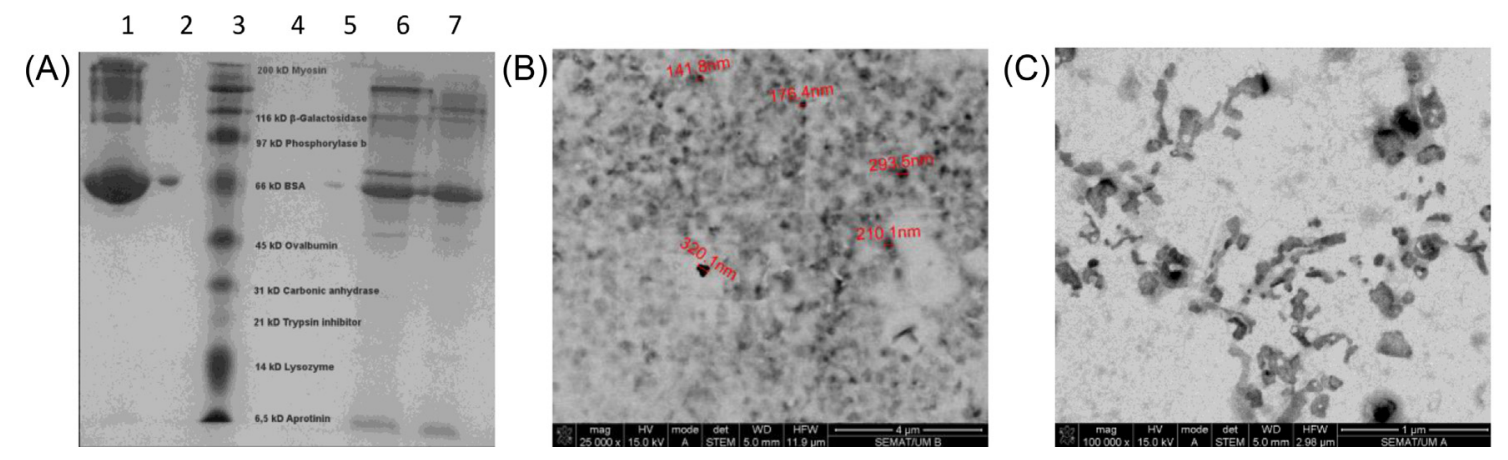

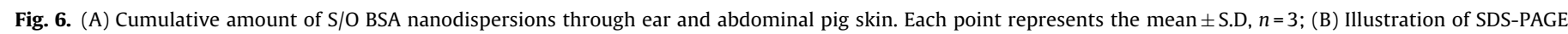

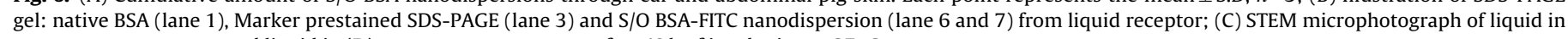
acceptor compartment and liquid in (D) receptor compartment, after $48 \mathrm{~h}$ of incubation at $37^{\circ} \mathrm{C}$.

The pig skin specimens were placed between two compartments of Franz cells: the donor and the receptor. Following an incubation period of $48 \mathrm{~h}$ at $37^{\circ} \mathrm{C}$, the pig skin was removed from the Franz diffusion cell and washed several times to remove any particles that had not permeated the skin. The samples were then prepared for histological studies and the permeation results are presented as confocal laser microscopy images. The penetration extent and localization of BSA nanodispersions are depicted in Fig. 5 which is divided into two parts comprising $2 \mathrm{D}$ and $3 \mathrm{D}$ images respectively. Each image is the result of image acquisition at multiple times per second, allowing analysis of lateral diffusion kinetics. It is clear from confocal images that the fluorescence intensity of BSA nanodispersions is much higher at the SC and epidermis and diminishes as it crosses the skin to the dermis. These permeation results were achieved mainly due to the low average diameter of the particles due to their coating with a hydrophobic surfactant and dispersal in the IPM. This oil is reported to act upon the SC by liquefying the lamellar-gel phase of the SC lipids and possibly partially dissolving them [41]. IPM is also able to dissolve considerable amounts of cholesterol, which may act as a membrane stabilizer [42] and thereby increase the diffusivity within the SC and/or the partition coefficient between the SC and the vehicle of both the drug and the solvent [43]. Furthermore, IPM can be used as an emulsifier and as a co-solvent with skin penetration enhancement properties [44].

The rate-controlling step of penetration into the skin is known to be the traversing of the SC, the hydrophobic part of the skin.[11,45]
Quantitative estimation of cumulative nanodispersions permeation was calculated according to the equation described by Piao et al. [13] Fig. 6A presents the cumulative amount of permeated nanodispersions per area of surface for ear and abdominal pig skin. Based on Fig. 6A S/O nanodispersion readily cross the SC and after $2 \mathrm{~h}$ it was detected in the receiver solution which meant that it had effectively crossed the skin. The observed penetration increased with time until the $48 \mathrm{~h}$ of diffusion. The permeation levels obtained for the ear part were higher relative to the abdominal part due to the lesser thickness of the former. SDS-PAGE gel electrophoresis (Fig. 6B) revealed the presence of the protein in the receiver compartment, confirming that it had efficiently crossed the skin barrier. The protein detected by electrophoresis corresponded to a solution containing spherical or/and crumble particles resulting from the passage of several barriers along the different skin layers. This result was corroborated by STEM images (Fig. 6D) which showed an irregular nanodispersion of spherical integral small particles and disintegrated larger particles. The total amount of the initial nanodispersion subjected to diffusion was not able to cross the SC due to inherent method conditions that induce turbulence to the solution (Fig. 6C). Moreover, the initial solution was composed of particles of large sizes (Fig. 4B) that had tendency to aggregate and disintegrate when forced to diffuse by gravity.

As previously reported, we prepared our nanodispersions based on Tahara et al. methodology. However several parameters were modified and improved, such as the type of homogenization, the structure of sucrose ester, the solvent and the sonication step. 

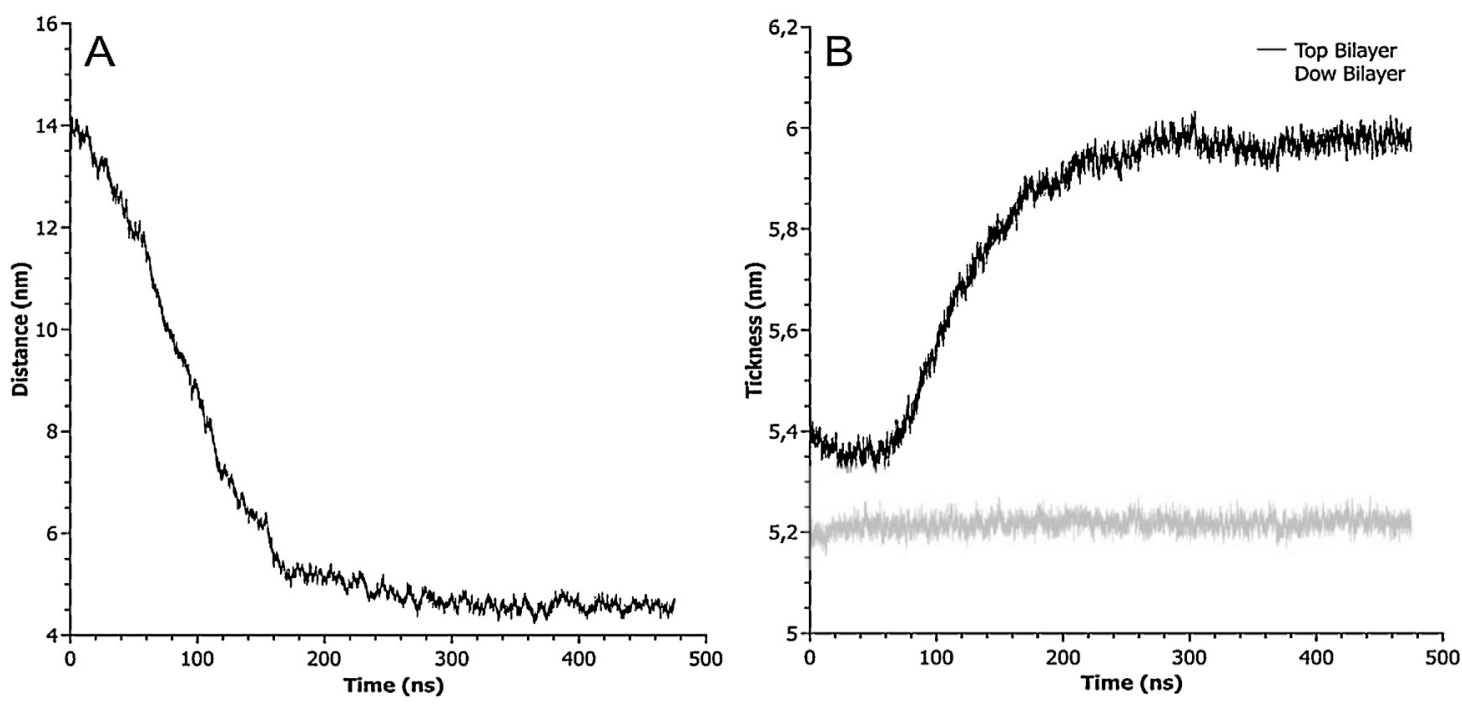

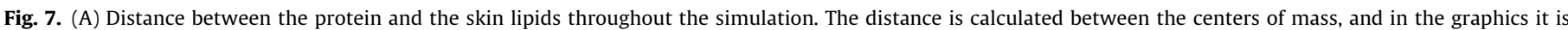

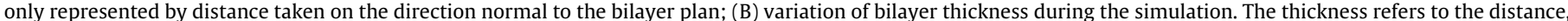
measured on a direction normal to the bilayer plan, between the centers of mass of the sulfate groups from cholesterol sulfate.

Comparing both set of results we were able to achieve much lower particle sizes and polydispersity indexes. Moreover, an efficient transdermal perfusion was attained for $60 \mathrm{kDa}$ Albumin-FITC nanodispersions ( $160 \mathrm{~nm}$ ) while Tahara et al. only achieved satisfactory perfusion with $6 \mathrm{kDa}$ preparations.

\subsection{Molecular dynamics simulations}

In order to fully understand the overall mechanism of skin perfusion for this protein/surfactant/oil complex the theoretical studies using Molecular Dynamics Simulations were performed. The permeation studies previously described were performed on pig skin, but these simulations were performed using the human skin model.

Giving the complexity of CERs on SC, any representation of the skin will be an approximation, thus for representing the CERs fraction of SC, CER(NS) was chosen mainly because it has been used before by others in Molecular Dynamics Simulations of SC [22,46-48]. CER(NS) corresponds to a ceramide with one nonhydroxy fatty acid and one sphingosine moiety, specifically a C24 fatty acid moiety and a C16 sphingosine were used. The free fatty acid fraction was considered to be exclusively made by lignoceric acid. The relative amounts of the constituents of SC used in the simulations were chosen to reflect the composition of a typical young human skin [49].

The Molecular Dynamics Simulations were made using the Gromacs [16] package and using the coarse grained force field Martini[17]. The lipids were arranged as a double bilayer using CELL microcosmos [20], with an initial size of $43 \mathrm{~nm} \times 43 \mathrm{~nm} \times 32 \mathrm{~nm}$, including the water molecules and the protein. The simulations, 5 replicas with different starting velocities taken from a MaxwellBoltzman distribution, were extended to $475 \mathrm{~ns}$. At this timepoint, the system reached a stable configuration, as can be observed in Fig. 7 and Fig. 8. The simulations were extended at least to $900 \mathrm{~ns}$, and it was not possible to observe translocation of the nanoparticle between lipid bilayers.

Fig. 7A represents the distance between the protein and the skin lipids from both layers. In the first 180 ns the distance drops rapidly and then it stabilized. From 300 ns onwards it is not possible to observe any major changes in this distance. A similar behavior can be observed in Fig. 7B, in which the thickness of each bilayer is depicted. The thickness of the lower bilayer remained unchanged throughout the course of the simulation. The behavior of the top bilayer however, is directly related to the distance between the protein and the skin lipids. From Fig. 7B it is clear that, within these time scales, the protein only distorts the top layer.

The relative position of the protein with respect to the bilayers was determined by the density profiles across the simulations box. These density profiles are represented in Fig. 8 for the initial and the final system configurations following $475 \mathrm{~ns}$ of simulation. Initially the protein and the skin lipids were completely separated, but after $475 \mathrm{~ns}$, it is possible to observe that the protein is integrated into the top bilayer, as demonstrated by the density maximum reading at $12.5 \mathrm{~nm}$.

The evolution of the system throughout the simulation is represented in Fig. 9. Initially the protein microsphere is not interacting with the top bilayer, but it is rapidly engulfed by the lipids. As already demonstrated by Fig. 7, the system reached a stable configuration after approximately $200 \mathrm{~ns}$.

The data generated by the simulations revealed the importance of each component in the mixture. IPM plays a major role in the interaction of the emulsion with the lipids, while the surfactant is

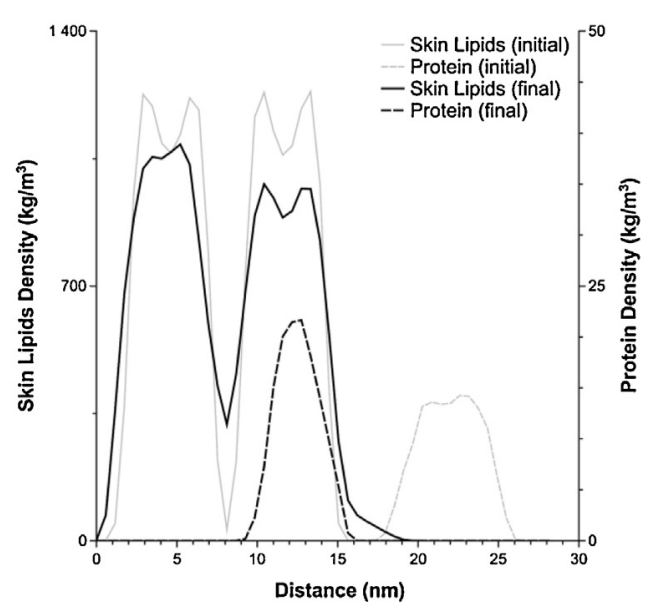

Fig. 8. Density profiles for the skin lipids and protein at the beginning and the end of the simulation. The distances were measured in the direction normal to the lipidic bilayer. 


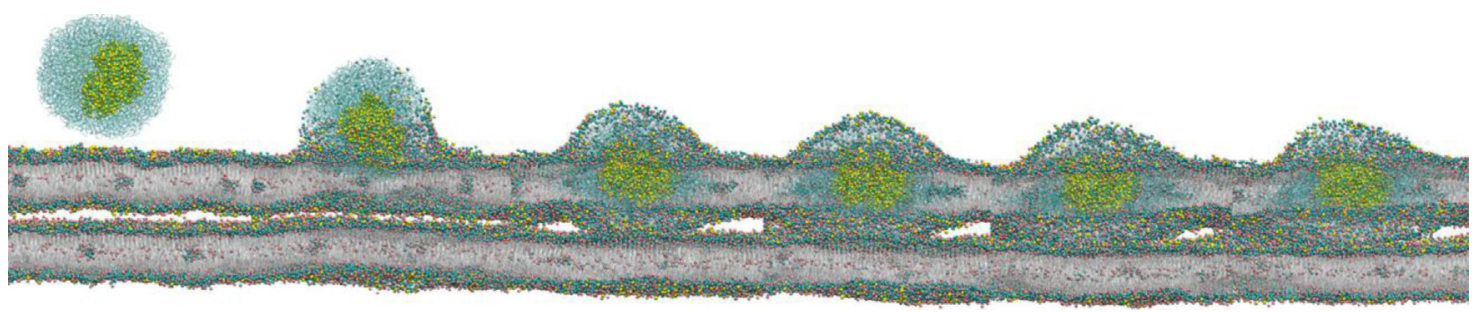

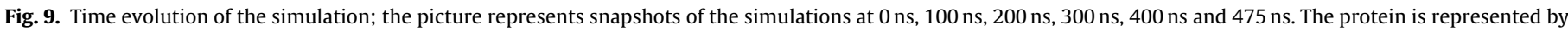

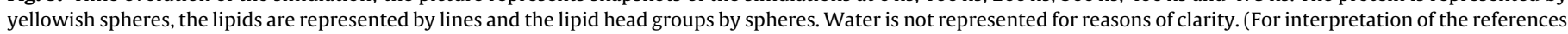
to color in this figure legend, the reader is referred to the web version of this article.)

responsible for the solubility of the hydrophilic protein within the oil.

\section{Conclusions}

We have experimentally demonstrated that high molecular weight proteins coated with hydrophobic surfactant molecules can effectively permeate the skin. These results provide valuable scientific data for the design of new protein delivery systems. The ability to deliver large proteins transdermally across a hitherto impervious membrane is also of value to the biopharmaceutical and cosmetics industries.

\section{Acknowledgments}

We thank Matadouro - Central Carnes de Entre Douro e Minho, Lda for their support on pig samples. The histological studies were supported by the Department of Histology from Life and Health Sciences Research Institute (ICVS), University of Minho. The confocal 3D images were performed by Department of Chemistry and Kanbar Laboratory for Nanomaterials Bar-Ilan University Center for Advanced Materials and Nanotechnology. Carla Silva would like to thanks Fundação para a Ciência e a Tecnologia for their funding (SFRH/BPD/46515/2008).

\section{References}

[1] G. Cevc, U. Vierl, Nanotechnology and the transdermal route: a state of the art review and critical appraisal, J. Control. Release 141 (2010) 277-299.

[2] R. Richert, A. Schrader, K. Schrader, Transdermal delivery of two antioxidants from different cosmetic formulations, Int. J. Cosmet. Sci. 25 (2003).

[3] M.R. Prausnitz, R. Langer, Transdermal drug delivery, Nat. Biotechnol. 26(2008) 1261-1268.

[4] P. Karande, A. Jain, K. Ergun, V. Kispersky, S. Mitragotri, Design principles of chemical penetration enhancers for transdermal drug delivery, Proc. Natl. Acad. Sci. U.S.A. 102 (2005) 4688-4693.

[5] R.J. Scheuplein, I.H. Blank, Permeability of the skin, Physiol. Rev. 51 (1971) 702-747.

[6] Y. Masukawa, H. Narita, E. Shimizu, N. Kondo, Y. Sugai, T. Oba, R. Homma, J. Ishikawa, Y. Takagi, T. Kitahara, Y. Takema, K. Kita, Characterization of overall ceramide species in human stratum corneum, J. Lipid Res. 49 (2008) 1466-1476.

[7] Y. Masukawa, H. Narita, H. Sato, A. Naoe, N. Kondo, Y. Sugai, T. Oba, R. Homma, J. Ishikawa, Y. Takagi, T. Kitahara, Comprehensive quantification of ceramide species in human stratum corneum, J. Lipid Res. 50 (2009) 1708-1719.

[8] R. t'Kindt, L. Jorge, E. Dumont, P. Couturon, F. David, P. Sandra, K. Sandra, Profiling and characterizing skin ceramides using reversed-phase liquid chromatography-quadrupole time-of-flight mass spectrometry, Anal. Chem. 84 (2012) 403-411.

[9] L. Norlén, I. Nicander, A. Lundsjö, T. Cronholm, B. Forslind, A new HPLC-based method for the quantitative analysis of inner stratum corneum lipids with special reference to the free fatty acid fraction, Arch. Dermatol. Res. 290 (1998) 508-516.

[10] M.J. King, I. Badea, J. Solomon, P. Kumar, J.K. Gaspar, M. Foldvari, Transdermal delivery of insulin from a novel biphasic lipid system in diabetic rats, Diab. Technol. Ther. 4 (4) (2002) 479-488.

[11] Y. Tahara, S. Honda, N. Kamiya, H. Piao, A. Hirata, E. Hayakawa, T. Fujii, M. Goto, A solid-in-oil nanodispersion for transcutaneous protein delivery, J. Control. Release 131 (2008) 14-18.
[12] D. Pissuwan, K. Nose, R. Kurihara, K. Kaneko, Y. Tahara, N. Kamiya, M. Goto, Y. Katayama, T. Niidome, A solid-in-oil dispersion of gold nanorods can enhance transdermal protein delivery and skin vaccination, Small 7 (2011) 215-220.

[13] H. Piao, N. Kamiya, A. Hirata, T. Fujii, M. Goto, A novel solid-in-oil nanosuspension for transdermal delivery of diclofenac sodium, Pharm. Res. 25 (2007) 896-901.

[14] H. Yoshiura, M. Hashida, N. Kamiya, M. Goto, Factors affecting protein release behavior from surfactant-protein complexes under physiological conditions, Int. J. Pharm. 338 (2007) 174-179.

[15] O.H. Lowry, N.J. Rosebrough, A.L. Farr, R.J. Randall, Protein measurement with the Folin phenol reagent, J. Biol. Chem. 193 (1) (1951) 265-275.

[16] B. Hess, C. Kutzner, D. van der Spoel, E. Lindahl, GROMACS algorithms for highly efficient, load-balanced, and scalable molecular simulation, J. Chem. Theory Comput. 4 (2008) 435-447.

[17] S.J. Marrink, H.J. Risselada, S. Yefimov, D.P. Tieleman, A.H. de Vries, The MARTINI force field: coarse grained model for biomolecular simulations, J. Phys. Chem. B 111 (2007) 7812-7824.

[18] B. Hess, H. Bekker, H.J.C. Berendsen, J.G.E.M. Fraaije, LINC.S., A linear constraint solver for molecular simulations, J. Comput. Chem. 18 (1997) $1463-1472$.

[19] H.J.C. Berendsen, J.P.M. Postma, W.F. van Gunsteren, A. DiNola, J.R. Haak, Molecular dynamics with coupling to an external bath, J. Chem. Phys. 81 (1984) 3684.

[20] B. Sommer, T. Dingersen, C. Gamroth, S.E. Schneider, S. Rubert, J. Kruger, K.-J. Dietz, CELLmicrocosmos 2.2 MembraneEditor: a modular interactive shapebased software approach to solve heterogeneous membrane packing problems, J. Chem. Inform. Model. 51 (2011) 1165-1182.

[21] J.T. Huzil, S. Sivaloganathan, M. Kohandel, M. Foldvari, I. Kotsireas, R. Melnik, B. West, Modeling the effects of lipid composition on stratum corneum bilayers using molecular dynamics simulations, AIP Conf. Proc. 1368 (2011) 175-178.

[22] M.I. Hoopes, M.G. Noro, M.L. Longo, R. Faller, Bilayer structure and lipid dynamics in a model stratum corneum with oleic acid, J. Phys. Chem. B 115 (2011) 3164-3171.

[23] W.S. Lee, Integral hair lipid in human hair follicle, J. Dermatol. Sci. 64 (2011) 153-158.

[24] R. Silva, H. Ferreira, N.G. Azoia, U. Shimanovich, G. Freddi, A. Gedanken, A. Cavaco-Paulo, Insights on the mechanism of formation of protein microspheres in a biphasic system, Mol. Pharm. 9 (2012) 3079-3088.

[25] S. Gopalakrishnana, R. Sujathaa, Ageing studies of cardanol based polyurethanes, Res. J. Pharm. Biol. Chem. Sci. 2 (2011).

[26] M. Blasco, J. García-Reverter, E. Casas, E. Riera, Effect of high power ultrasounds on mass-transfer zone in supercritical fluid extraction processes, in: Proceedings of 20th International Congress on Acoustics, 2010.

[27] K.S. Durbha, K. Aravamudan, Quantification of surface area and intrinsic mass transfer coefficient for ultrasound-assisted dissolution process of a sparingly soluble solid dispersed in aqueous solutions, Ultrason. Sonochem. 19 (2012) 509-521.

[28] J. Rae, M. Ashokkumar, O. Eulaerts, C. von Sonntag, J. Reisse, F. Grieser, Estimation of ultrasound induced cavitation bubble temperatures in aqueous solutions, Ultrason. Sonochem. 12 (2005) 325-329.

[29] F. Marzulli, H.I. Maibach, Relevance of animal models: the hexachlorophene story, Maibach, H.I., New York, 1975.

[30] R.L. Bronaugh, R.F. Stewart, E.R. Congdon, Methods for in vitro percutaneous absorption studies II. Animal models for human skin, Toxicol. Appl. Pharmacol. 62 (1982) 481-488.

[31] J.M. Haigh, E.W. Smith, The selection and use of natural and synthetic membranes for in vitro diffusion experiments, Eur. J. Pharm. Sci. 2 (1994) 311-330.

[32] G.M. Morris, J.W. Hopewell, Epidermal cell kinetics of the pig: a review, Cell Prolif. 23 (1990) 271-282.

[33] T.P. Sullivan, W.H. Eaglstein, S.C. Davis, P. Mertz, The pig as a model for human wound healing, Wound Repair Regen. 9 (2001) 66-76.

[34] N. Harunari, K.Q. Zhu, R.T. Armendariz, H. Deubner, P. Muangman, G.J. Carrougher, F.F. Isik, N.S. Gibran, L.H. Engrav, Histology of the thick scar on the female, red Duroc pig: final similarities to human hypertrophic scar, Burns 32 (2006) 669-677.

[35] S.A. Gallo, A. Sen, M.L. Hensen, S.W. Hui, Time-dependent ultrastructural changes to porcine stratum corneum following an electric pulse, Biophys. J. 76 (1999) 2824-2832. 
[36] K.A. Holbrook, G.F. Odland, Regional differences in the thickness (cell layers) of the human stratum corneum: an ultrastructural analysis, J. Invest. Dermatol. 62 (1974) 415-422.

[37] R.C. Wester, J. Melendres, L. Sedik, H. Maibach, J.E. Riviere, Percutaneous absorption of salicylic acid, theophylline, 2.4-dimethylamine, diethyl hexyl phthalic acid, andp-aminobenzoic acid in the isolated perfused porcine skin flap compared to manin vivo, Toxicol. Appl. Pharmacol. 151 (1998) 159-165.

[38] S. Singh, K. Zhao, J. Singh, In vitro permeability and binding of hydrocarbons in pig ear and human abdominal skin drug and chemical, Toxicology 25 (2002) 83-92.

[39] N. Sekkat, Y.N. Kalia, R.H. Guy, Porcine ear skin as a model for the assessment of transdermal drug delivery to premature neonates, Pharm. Res. 21 (2004) 1390-1397.

[40] G.A. Simon, H.I. Maibach, The pig as an experimental animal model of percutaneous permeation in man: qualitative and quantitative observations - an overview, Skin Pharmacol. Appl. Skin Physiol. 15 (2000) 229-234.

[41] A. Arellano, S. Santoyo, C. Martǐn, P. Ygartua, Influence of propylene glycol and isopropyl myristate on the in vitro percutaneous penetration of diclofenac sodium from carbopol gels, Eur. J. Pharm. Sci. 7 (1999) 129-135.

[42] C.S. Leopold, B.C. Lippold, An attempt to clarify the mechanism of the penetration enhancing effects of lipophilic vehicles with differential scanning calorimetry (DSC), J. Pharm. Pharmacol. 47 (4) (1995) 276-281.
[43] K. Sato, K. Sugibayashi, Y. Morimoto, Effect and mode of action of aliphatic esters on the in vitro skin permeation of nicorandil, Int. J. Pharm. 43 (1988) 31-40.

[44] P. Klaffenbach, D. Kronenfeld, Analysis of impurities of isopropyl myristate by gas-liquid chromatography, J. Chromatogr. A 767 (1997) 330-334.

[45] K. Moser, K. Kriwet, A. Naik, Y.N. Kalia, R.H. Guy, Passive skin penetration enhancement and its quantification in vitro, Eur. J. Pharm. Biopharm. 52 (2001) 103-112.

[46] R. Notman, J. Anwar, Breaching the skin barrier - insights from molecular simulation of model membranes, Adv. Drug Deliv. Rev. (2012).

[47] R. Notman, W.K. den Otter, M.G. Noro, W.J. Briels, J. Anwar, The permeability enhancing mechanism of DMSO in ceramide bilayers simulated by molecular dynamics, Biophys. J. 93 (2007) 2056-2068.

[48] C. Das, M.G. Noro, P.D. Olmsted, Simulation studies of stratum corneum lipid mixtures, Biophys. J. 97 (2009) 1941-1951.

[49] V. Schreiner, G.S. Gooris, S. Pfeiffer, G. Lanzendorfer, H. Wenck, W. Diembeck, E. Proksch, J. Bouwstra, Barrier characteristics of different human skin types investigated with X-ray diffraction, lipid analysis, and electron microscopy imaging, J. Invest. Dermatol. 114 (2000) 654-660. 